\title{
Colorimetric Signaling of Water Content in Acetonitrile by Phenolic Dye-Fluoride Complexes
}

\author{
Jung Ok Moon, Youn Hwan Kim, Myung Gil Choi, and Suk-Kyu Chang* \\ Department of Chemistry, Chung-Ang University, Seoul 156-756, Korea. *E-mail: skchang@cau.ac.kr \\ Received June 27, 2011, Accepted July 27, 2011
}

Key Words : Water content, Acetonitrile, Phenolic dye-fluoride complex, Colorimetry, Ratiometry

Precisely determining and controlling the water content of chemicals and other commercial products are important considerations in multiple fields of chemistry and industrial processes. ${ }^{1}$ The water content of various commercial products including organic solvents is routinely determined by Karl Fischer titration or gas chromatography; ${ }^{2}$ however, alternative optical methods are quite desirable in terms of convenience and instrument requirements. Selective and sensitive optical signaling systems that utilize multifunctional dye molecules, ${ }^{3}$ such as merocyanines, ${ }^{4}$ flavones, ${ }^{5}$ chalcone, ${ }^{6} 3$-hydroxychromone, ${ }^{7}$ naphthalimide, ${ }^{8}$ and indole derivatives $^{9}$ have been developed as probes to sense the water content of commonly used organic solvents.

There are a variety of sophisticated fluoride sensors that are based on the molecular framework of hydrazones, thioureas, and amide derivatives. ${ }^{10}$ Other representative fluoride sensors are constructed from various molecular structures including silyl ether of resorufin, ${ }^{11}$ naphthylimides, ${ }^{12}$ and Lewis acidic boranes. ${ }^{13}$ Phenolic dyes are well-known to selectively interact with fluoride ions by hydrogen bonding, and azophenol derivatives in particular have been developed as fluoride-selective chromogenic sensors. ${ }^{14}$ Azophenol appended (thia)calix[4]arenes which function by complexation induced extended conjugation were used for colorimetric sensing of fluorides. ${ }^{15} \mathrm{Ru}(\mathrm{II})$ based polypyridyl complexes with pendant phenol or catechol groups can act as colorimetric sensors for fluoride ion. ${ }^{16}$ In addition, a series of polymers based on polyphenylenes that contain phenol-substituted oxadiazole moieties were prepared for chemosensing of fluoride ion. ${ }^{17}$

However, because the interaction between phenolic dyes and fluoride ions is through hydrogen bonding, recognition and/or sensing of such anions has usually been constrained to noncompetitive organic solvents such as acetonitrile and DMSO since aqueous solutions provide strongly competitive hydrogen bonding with water molecules. ${ }^{18}$ Recently, we reported a colorimetric system for the signaling of water content in organic solvents based on hydrazone-acetate complexes. ${ }^{19}$ In this paper, we have designed another convenient signaling system to determine the water content of acetonitrile, which utilizes the disruptive effect of water on complexation of phenolic dyes with fluoride ion. The variation of water content in the organic solvent was sensitively visualized by ratiometry as well as naked-eye detectable color changes.

Our strategy for developing a signaling system for analyzing the water content of acetonitrile was based on the effect of water on the well-known complex formation between phenols and fluoride ion in organic solvents. The readily available azo phenol and naphthol dyes 4-(4-nitrophenylazo)-1-naphthol (1) and Disperse Yellow 7 (2) were chosen to examine the potential for chromogenic signaling of water content (Scheme 1). Acetonitrile solutions of these dyes in the presence of fluoride ion exhibited quite pronounced chromogenic behavior, with changes in color from blue to yellow or orange in response to variations in water content.

UV-vis spectrum of $\mathbf{1}$ displayed a strong absorption band at $455 \mathrm{~nm}$ in acetonitrile. Upon treatment with increasing amounts of fluoride ion (as the tetrabutylammonium salt), the absorption spectrum of $\mathbf{1}$ changed significantly; the absorption band at $455 \mathrm{~nm}$ decreased, while a new strong band at $634 \mathrm{~nm}$ developed (Figure 1) with a concomitant solution color change from yellow to blue. This chromogenic behavior is known to result from hydrogen bonding of the phenol moiety of 1 with fluoride ion (Scheme 2). As shown in the right inset of Figure 1, constant absorbances at 455 and $634 \mathrm{~nm}$ were observed with 2 equiv of fluoride ion as has been reported with other phenol-fluoride sensor systems. ${ }^{16}$ A Job's plot for the 1-F system also suggested that the complexation stoichiometry for 1 with fluoride was 1:2 (left inset of Figure 1).

Next, the effect of water on the 1-F complex in acetonitrile
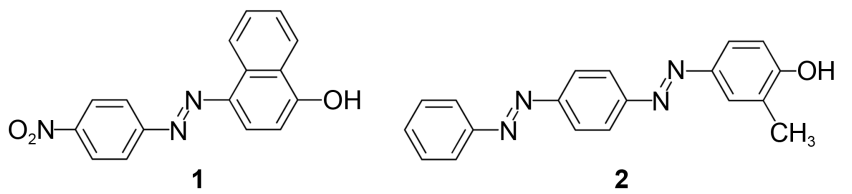

Scheme 1. Structures of 4-(4-nitrophenylazo)-1-naphthol (1) and Disperse Yellow 7 (2).

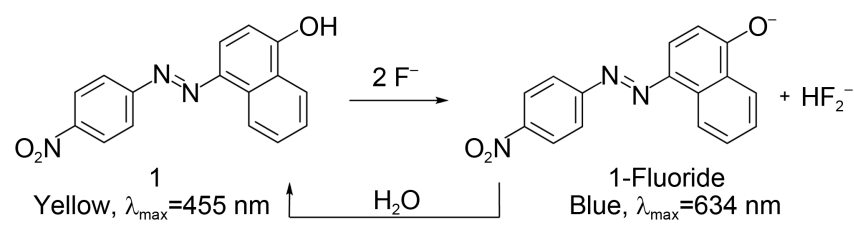

Scheme 2. Interaction of $\mathbf{1}$ with fluoride ions and potential use of the 1-F system for water signaling. 


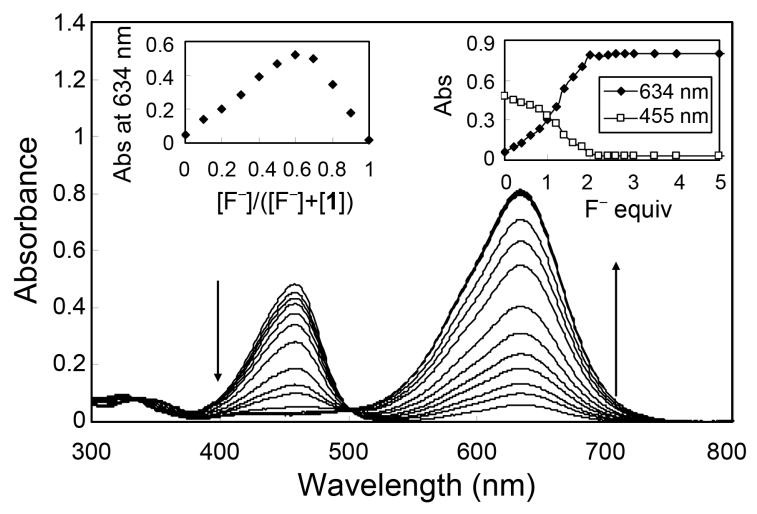

Figure 1. UV-vis spectra of $\mathbf{1}$ alone and in the presence of varying amounts of fluoride in acetonitrile. [1] $=1.0 \times 10^{-5} \mathrm{M}$. Left inset: Job's plot of the 1-F system using the absorbance at $634 \mathrm{~nm}$. Right inset: changes in the absorbances at 455 and $634 \mathrm{~nm}$ as a function of fluoride ion concentration.

was studied. As expected, the addition of $5 \%$ water to the 1F system $\left([1]=1.0 \times 10^{-5} \mathrm{M}\right.$ and $\left.\left[\mathrm{F}^{-}\right]=5.0 \times 10^{-5} \mathrm{M}\right)$ resulted in a UV-vis spectrum that was almost identical to 1 alone. This transformation is due to competition or breaking of the hydrogen bonding interaction between 1 and fluoride ions by water molecules (Scheme 2). The chromogenic response of 1-F to changes in water content suggests the complex could be useful as a visible detection probe for analyzing the water content of acetonitrile.

Based on this observation, a systematic investigation of the signaling of water content in acetonitrile was carried out. Changes in the absorption behavior of a 1-F system, prepared by adding 5 equiv of fluoride ion to $1\left(1.0 \times 10^{-5} \mathrm{M}\right)$, as a function of water content in acetonitrile were measured. Although, the signaling sensitivity was somewhat lower with excess fluoride, the uncertainty introduced by using exactly 2 equiv of fluoride for the assay would be mitigated. As the water content in acetonitrile increased, the absorption behavior progressively shifted from the 1-F spectrum to that of 1 alone (Figure 2), with the maximum wavelength of the longer absorption band around $634 \mathrm{~nm}$ steadily blue shifted. Concomitant with the spectral changes, the solution color progressively changed from blue to yellow, which was easily

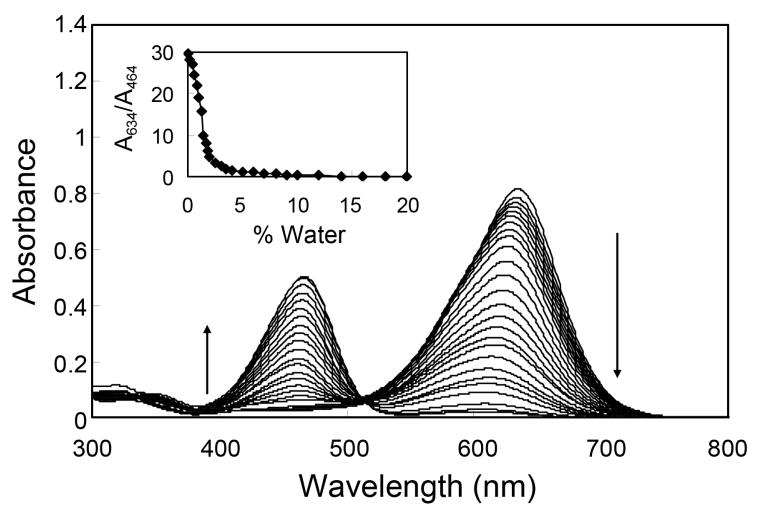

Figure 2. Changes in the UV-vis spectra of the 1-F system as a function of water content in acetonitrile. $[\mathbf{1}]=1.0 \times 10^{-5} \mathrm{M},\left[\mathrm{F}^{-}\right]=$ $5.0 \times 10^{-5} \mathrm{M}$. Inset: changes in $A_{634} / A_{464}$ as a function of $\%$ water. observable with the naked eye.

Changes in the absorption spectrum of the 1-F system as a function of water content could be conveniently analyzed by ratiometry using the absorbances at 634 and $464 \mathrm{~nm}$. A plot of $A_{634} / A_{464}$ as a function of water content gave a wellcorrelated relationship (inset of Figure 2). The ratio significantly decreased from approximately 30 for $100 \%$ acetonitrile to 1.2 for $5 \%$ water, then slowly decreased further to 0.3 for $10 \%$ water content. In particular, changes in the lower water content region $(<2 \%)$ were more pronounced, such that a ratiometric calibration curve could be constructed for determining a water content of less than $2 \%$ in acetonitrile. From this plot, the limit of detection for water content in acetonitrile with the 1-F system was determined to be $0.17 \%$.

Disperse Yellow 2 displayed similar chromogenic signaling behavior. Dye 2 had a strong absorption band at $380 \mathrm{~nm}$ in acetonitrile, which upon treatment with increasing amounts of fluoride ion decreased while a new strong band at $584 \mathrm{~nm}$ developed (Figure 3). Concomitant with the spectral changes, the solution color changed from orange to blue. As shown in the inset of Figure 3, constant absorbances at 380 and $584 \mathrm{~nm}$ were observed with approximately 2 equiv of fluoride ion. A Job's plot for the 2-F system suggested that the stoichiometry for complex $\mathbf{2}$ with fluoride was 1:2 (left inset of Figure 3).

The effect of water on the 2-F system in acetonitrile was also studied. Changes in absorption behavior of a $2-\mathrm{F}$ system, prepared by adding 5 equiv of fluoride ion to 2 ([2] $\left.=1.0 \times 10^{-5} \mathrm{M}\right)$ as a function of water content in acetonitrile were measured. As the water content increased, the absorption spectra progressively shifted from the spectrum of the 2-F system to that of $\mathbf{2}$ alone (Figure 4). From this titration result, the limit of detection for water content in acetonitrile with complex 2 was calculated to be $0.16 \%$.

The absorption maximum of the longer wavelength region of the 1-F and 2-F systems steadily shifted to shorter wavelength with increasing water content (Figure 5). The $\lambda_{\max }$ of the 1-F system was $634 \mathrm{~nm}$ in pure acetonitrile,

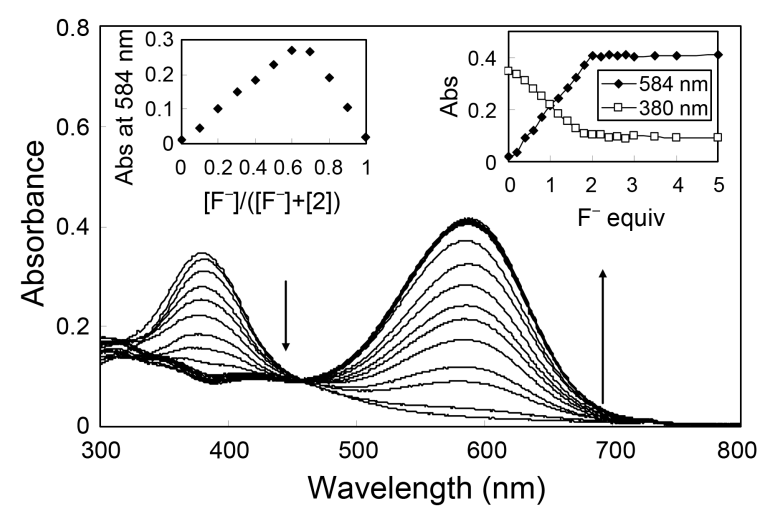

Figure 3. UV-vis spectra of $\mathbf{2}$ alone and in the presence of varying amounts of fluoride in acetonitrile. [2] $=1.0 \times 10^{-5} \mathrm{M}$. Left inset: Job's plot of the 2-F system using the absorbance at $584 \mathrm{~nm}$. Right inset: changes in the absorbances at 380 and $584 \mathrm{~nm}$ as a function of fluoride ion concentration. 


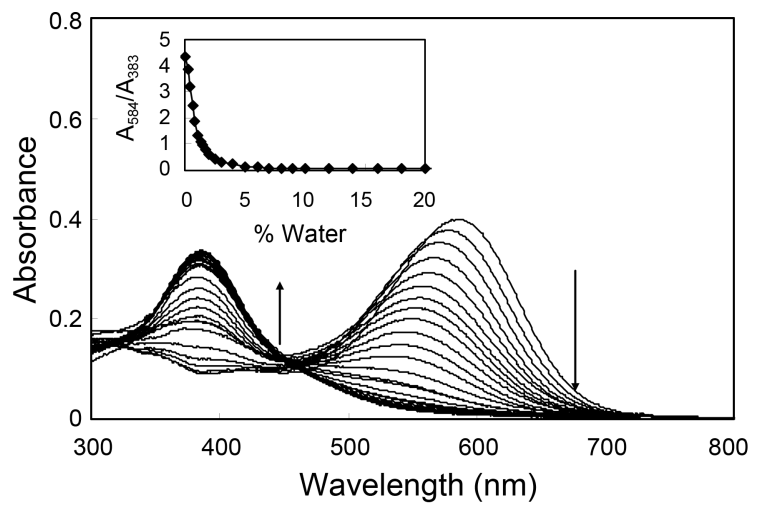

Figure 4. UV-vis titration of the $2-\mathrm{F}$ system with water in acetonitrile. [2] $=1.0 \times 10^{-5} \mathrm{M},\left[\mathrm{F}^{-}\right]=5.0 \times 10^{-5} \mathrm{M}$. Inset: changes in $A_{584} / A_{383}$ as a function of $\%$ water.

which upon addition of incremental amounts of water up to $15 \%$ significantly shifted to $608 \mathrm{~nm}(\Delta \lambda=-26 \mathrm{~nm})$. For higher concentrations of water, the absorption maximum could not be accurately determined due to significant broadening of the absorption band. The blue shift is due to stabilization of the phenoxide in the 1-F complex system in more polar, water rich media. ${ }^{20}$ Changes in the maximum absorption wavelength of the 1-F system in response to variations of water content provided a convenient tool for determining the water content over a wider concentration range up to $15 \%$ water. With the $2-\mathrm{F}$ system, changes in the maximum wavelength of the longer absorption band were more pronounced. The maximum absorbance around 584 $\mathrm{nm}$ steadily blue shifted to $532 \mathrm{~nm}(\Delta \lambda=-52 \mathrm{~nm})$ as the water content increased up to $3 \%$. At greater water concentrations, the maximum wavelength could not be determined with reasonable accuracy due to the broadened spectra. Changes in the absorption wavelength maximum of the 2-F system in response to variations of water content provided a convenient tool for analyzing up to $3 \%$ water in acetonitrile.

In summary, a simple chromogenic signaling system for the water content of acetonitrile based on phenolic dyefluoride complexes was developed. Signaling was dependent on competition of water molecules with the phenolic
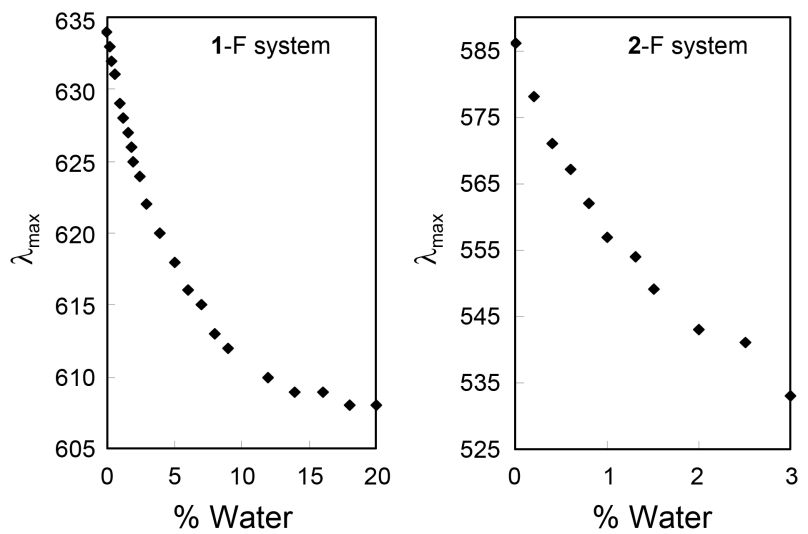

Figure 5. Changes in the absorption maximum of the longer wavelength region of the $1-\mathrm{F}$ and $2-\mathrm{F}$ systems as a function of water content in acetonitrile. $[\mathbf{1}]=[2]=1.0 \times 10^{-5} \mathrm{M},\left[\mathrm{F}^{-}\right]=5.0 \times 10^{-5} \mathrm{M}$. hydroxyl group of the dye for hydrogen bonding with fluoride ion. Pronounced chromogenic behavior, which was visible with the naked eye, was observed with the phenolic dye-fluoride systems. Changes in the absorption behavior were successfully analyzed by ratiometry as well as by shifts in the absorption maximum, and signals well for less than $3 \%$ water in acetonitrile. This phenolic dye-fluoride system could be useful as a convenient colorimetric and ratiometric probe for analyzing the water content of acetonitrile.

\section{Experimental Section}

General. 4-(4-Nitrophenylazo)-1-naphthol, Disperse Yellow 7 , and tetrabutylammonium fluoride were purchased from Sigma-Aldrich Co. and were used without further purification. UV-vis spectra were measured with a Jasco V-550 spectrophotometer equipped with a Peltier temperature controller. Anhydrous grade acetonitrile was obtained from Sigma-Aldrich Co.

Titration of Dyes with Fluoride Ion. Stock solutions of 1, 2, and tetrabutylammonium fluoride in acetonitrile were prepared. Aliquots of the dye and fluoride stock solutions were combined and then diluted with acetonitrile to a volume of $3.0 \mathrm{~mL}$. The final concentrations of the dyes and fluoride were $1.0 \times 10^{-5} \mathrm{M}$ and $0 \sim 5.0 \times 10^{-5} \mathrm{M}$ (5 equiv), respectively. UV-vis spectra of thoroughly mixed solutions were measured at $25^{\circ} \mathrm{C}$.

Measurement of Water Content in Acetonitrile. Aliquots of water were added to the acetonitrile solution of the dye-fluoride system, prepared by mixing solutions of dye $\mathbf{1}$ or 2 with 5 equiv of tetrabutylammonium fluoride, and the mixture was diluted with acetonitrile to a final volume of 3.0 $\mathrm{mL}$. The final concentrations of dye and fluoride were $1.0 \times$ $10^{-5} \mathrm{M}$ and $5.0 \times 10^{-5} \mathrm{M}$, respectively. Water content was increased up to $20 \%$, after which no detectable change was observed in the UV-vis spectra of the solution. The limit of detection for water content was determined by plotting the changes in absorbance versus $\log \left[\mathrm{H}_{2} \mathrm{O}\right]$ following the reported procedure. ${ }^{21}$

Acknowledgments. This research was supported by Chung-Ang University Research Scholarship Grants in 2011 (J.O.M.).

\section{References}

1. (a) Wang, Q.; Li, X.; Wang, L.; Cheng, Y.; Xie, G. Ind. Eng. Chem. Res. 2005, 44, 4518. (b) Al-Zuhair, S.; Jayaraman, K. V.; Krishnan, S.; Chan, W.-H. Biochem. Eng. J. 2006, 30, 212. (c) O'Keefe, W. K.; Ng, F. T. T.; Rempel, G. L. J. Chromatogr. A 2008, 1182, 113. (d) Nguyen, T. N. P.; Kim, K.-J. Ind. Eng. Chem. Res. 2010, 49, 4842.

2. (a) Fischer, K. Angew. Chem. 1935, 48, 394. (b) Kestens, V.; Conneely, P.; Bernreuther, A. Food Chem. 2008, 106, 1454.

3. (a) Hisamoto, H.; Manabe, Y.; Yanai, H.; Tohma, H.; Yamada, T.; Suzuki, K. Anal. Chem. 1998, 70, 1255. (b) Citterio, D.; Kawada, T.; Yagi, J.; Ishigaki, T.; Hisamoto, H.; Sasaki, S. I.; Suzuki, K. Anal. Chim. Acta 2003, 482, 19.

4. (a) Kumoi, S.; Kobayashi, H.; Ueno, K. Talanta 1972, 19, 505. (b) 
Lu, H.; Rutan, S. C. Anal. Chem. 1996, 68, 1381.

5. Liu, W.; Wang, Y.; Jin, W.; Shen, G.; Yu, R. Anal. Chim. Acta 1999, 383, 299

6. Niu, C. G.; Guan, A. L.; Zeng, G. M.; Liu, Y. G.; Li, Z. W. Anal. Chim. Acta 2006, 577, 264.

7. Ercelen, S.; Klymchenko, A. S.; Demchenko, A. P. Anal. Chim. Acta 2002, 464, 273.

8. Niu, C. G.; Qin, P. Z.; Zeng, G. M.; Gui, X. Q.; Guan, A. L. Anal. Bioanal. Chem. 2007, 387, 1067.

9. Kim, K. N.; Song, K. C.; Noh, J. H.; Chang, S.-K. Bull. Korean Chem. Soc. 2009, 30, 197.

10. Gunnlaugsson, T.; Glynn, M.; Tocci, G. M.; Kruger, P. E.; Pfeffer, F. M. Coord. Chem. Rev. 2006, 250, 3094.

11. Kim, S. K.; Hong, J.-I. Org. Lett. 2007, 9, 3109.

12. Gunnlaugsson, T.; Kruger, P. E.; Jensen, P.; Pfeffer, F. M.; Hussey, G. M. Tetrahedron Lett. 2003, 44, 8909.

13. Hudnall, T. W.; Gabbaï, F. P. J. Am. Chem. Soc. 2007, 129, 11978.

14. (a) Lee, D. H.; Lee, K. H.; Hong, J.-I. Org. Lett. 2001, 3, 5. (b)
Lee, D. H.; Lee, H. Y.; Lee, K. H.; Hong, J.-I. Chem. Commun. 2001, 1188. (c) Chen, C.-F.; Chen, Q.-Y. New J. Chem. 2006, 30, 143 .

15. Kumar, M.; Babu, J. N.; Bhalla, V. Talanta 2010, 81, 9.

16. Jose, D. A.; Kar, P.; Koley, D.; Ganguly, B.; Thiel, W.; Ghosh, H. N.; Das, A. Inorg. Chem. 2007, 46, 5576.

17. Zhou, G.; Cheng, Y.; Wang, L.; Jing, X.; Wang, F. Macromolecules 2005, 38, 2148

18. (a) Cho, E. J.; Ryu, B. J.; Lee, Y. J.; Nam, K. C. Org. Lett. 2005, 7 , 2607. (b) Cho, E. J.; Moon, J. W.; Ko, S. W.; Lee, J. Y.; Kim, S. K.; Yoon, J.; Nam, K. C. J. Am. Chem. Soc. 2003, 125, 12376.

19. Kim, Y. H.; Choi, M. G.; Im, H. G.; Ahn, S.; Shim, I. W.; Chang, S.-K. Dyes Pigm. in press.

20. Cysewski, P.; Szefler, B.; Szatyłowicz, H.; Krygowski, T. M. New J. Chem. 2009, 33, 831.

21. Shortreed, M.; Kopelman, R.; Kuhn, M.; Hoyland, B. Anal. Chem. 1996, 68, 1414 . 